\title{
THE INFLUENCE OF RELAXATION TRAINING TECHNIQUES ON PILOTS' LEVELS OF TOLERANCE TO MOTION SICKNESS SYMPTOMS DURING SIMULATED FLIGHT - PRELIMINARY RESULTS
}

\author{
Krzysztof KOWALCZUK', Michał JANEWICZ², Zofia PRZYMUS³, Tadeusz GRZESZUK', \\ Robert KILIAN', Stefan P. GAŹDZIŃSKI ${ }^{4}$ \\ 1 Department of Simulator Studies and Aeromedical Training, Military Institute of Aviation Medicine, \\ Warsaw, Poland \\ 2 SWPS University of Social Sciences and Humanities, Warsaw, Poland \\ 3 Kozminski University, Warsaw, Poland \\ 4 Creative Neuroscience Lab - CNS Lab, Military Institute of Aviation Medicine, Warsaw, Poland
}

Source of support: This project was supported by DNiSW MON and WIML via the "High-G" project nr 533/2016/DA.

Author's address: M. Janewicz, Military Institute of Aviation Medicine, Krasińskiego 54/56 Street, 01-755 Warsaw, Poland, e-mail: michal.janewicz@gmail.com

Introduction: We wanted to assess whether or not relaxation techniques may be useful tools to help pilots with habituation and desensitization, and if they could be used to prevent the symptoms of motion sickness?

Methods: We have decided to use The Motion Sickness Assessment Questionnaire (MSAQ). A MiG - 29 aircraft simulator was used during the testing procedure. The Schultz Autogenic Training procedure was used for relaxation training.

Results: Results: Statistically significant differences were found in the overall score $(0,0054 p<0,05)$ as in some of the factors: Central $(0,015 p<0,05)$ and Sopite-related $(0,035 p<0,05)$. Both the remaining factors exhibited a statistical trend. All the raw scores: overall and factoral ones were lower in the second testing in comparison with the first one.

Discussion: The results show us that a simple, low time-consuming and inexpensive method can possibly be used along other training options to further expand pilots' skills.

Keywords: pilots, relaxation training, motion sickness, simulated flight, air force, military 


\section{NTRODUCTION}

We wanted to assess whether or not relaxation techniques may be useful tools to help pilots with habituation and desensitization, and if they could be used to prevent the symptoms of motion sickness $[2,3]$ ? Autogenic training gives its user the possibility of achieving autonomic self-regulation. It serves its purpose by removing environmental distractions using a set of easy to learn, accessible exercises. Also, it may be used over a long period of time if needed. The Schultz Autogenic Training is a guided meditation technique in which a narrator's voice guides the user though the process. Autogenic training is based around a few main domains: muscle tension reduction, mental repetition of a verbal formula and passive concentration (the user is instructed to concentrate on inner sensations rather than environmental stimuli) all of which are set to relax both the psychological and the physiological domain [5].

The Schultz Autogenic Training that we utilized is usually performed while sitting or lying down in a comfortable, relaxed position. The technique consists of a few standard exercises:

1. Muscular relaxation: the user repeats a formula (either out loud or in one's thoughts) emphasizing heaviness. Usually something like: "My left hand is heavy" then "my left lower arm is heavy" and so on.

2. Passive concentration on feeling warmth: typically initiated by the instruction "My right/left arm is warm" or "I feel warmth in my arm" and continued all over the body.

3. Initiation of cardiac activity: typically using the "My heartbeat is calm and regular" formula [5].

Motion sickness should be understood as a group of individual symptoms. It starts when a person (a pilot in this case) is exposed to motion stimuli that they are not familiar with. Motion sickness has five main types depending on the type of vehicle it is associated with: sea sickness, car sickness, air sickness, simulator sickness and space sickness. The human centrifuge is a simulator that gives its operators the opportunity to fly with real G-loads, however just like any other simulator, it is not perfect, and sometimes causes motion sickness. Most of the symptoms of all of the above-mentioned motion sickness types are similar and are caused by the same mechanism. The names, motion sickness and i.e. air sickness can be used interchangeably. When there is any inconsistency in the information gathered from the visual and the vestibular system, symptoms may develop. If the information about the pilot's position gathered from the visual and the vestibular system is homogeneous, the symptoms of motion sickness will not occur. The severity of motion sickness may vary among people, individual susceptibility is the most important element in regard of the risk of developing sickness but it is also dependent on the type, strength and duration of the stimulus. The symptoms of motion sickness sum up to a substantially long list of problems which may occur: the main symptom of motion sickness is nausea, a sickness in the stomach, especially when accompanied by sitophobia and an involuntary impulse to vomit. The signs of nausea are: discomfort in the stomach, sweating, pallor, salivation and these all build up to vomiting. Usually, the earliest symptom is discomfort in the stomach. If the situation of uneasiness continues, pallor usually occurs and cold sweating begins. If the stimuli is not eliminated, the patient may experience increased salivation, bodily warmth and lightheadedness. At that point vomiting becomes a possibility [1].

\section{METHODS}

Firstly, we assessed which motion sickness questionnaire to use; which one of the possible choices is the most reliable, well established method to use in the specific setting of the human centrifuge. Then came the idea of introducing other types of training, such as relaxation techniques, to the pool of methods to help pilots with their tasks. The questionnaire is the most reliable, well established and easy to use method. We wanted to use the best questionnaire available for our needs. After a careful analysis and assessment of the available questionnaires, we have decided to use The Motion Sickness Assessment Questionnaire (MSAQ) [4]. Because of various levels of English proficiency in our test pilots, we have decided to use the translated version of MSAQ. We planned to test as many jet pilots as possible. During the given time, we tested 8. All the pilots agreed to participate. The test subjects were experienced in flight, all male, between 26-46 years old, active jet fighter pilots. They all had to hold a current, valid permission to fly. The Military Institute of Aviation Medicine ethics committee granted permission for the tests carried out in our unit. The procedure is:

1. First MSAQ testing right after the first test flight.

2. At least 3 weeks of at least 4 relaxation sessions per week before the next phase.

3. Second MSAQ testing right after the second test flight.

A MiG- 29 aircraft simulator was used during the testing procedure. The BFM (Basic Fighter Maneuvers: tactical movements performed by a fighter 
aircraft during air combat maneuvering) model consisted of:

1. Offensive maneuvers with limited parameters: $6 \mathrm{Gz}$ max, IAS (Indicated Air Speed) up to 400 knots or $740 \mathrm{~km} / \mathrm{h}$, same altitude flight. The maneuvers performed were: BREAK LEFT, BREAK RIGHT. The aircraft turns left / right, the maneuver consists of turning sharply across the attacker's flight path, to increase the AOT (angle off tail).

2. In the second part of the BFM: $\max G z$ 9, IAS 400500 Knots at the altitude of 5000-12000 feet. The maneuvers were: HARD BREAK LEFT, HARD BREAK RIGHT. The aircraft turns left / right with maximal flight parameters (speed, Gz, bank angle) with single circle fight, high aspect "butterfly" (the offensive aircraft makes a turn towards the opponent and defensive aircraft to the opposite side with $\max \mathrm{Gz}$ and bank angle, air combat maneuvering with head on position. After the pass, both fighters may turn to engage) and Yo-yo (variable altitude). Yo-Yo is one of the most useful maneuvers, which sacrifices altitude for an instantaneous increase in speed.

The MSAQ test is shown in its entirety above - we have used the Polish version as previously mentioned.

As for the Schultz Autogenic Training, the entirety of its instruction is in the audio file, together with the procedure. Because of the various English knowledge levels of our test pilots, we have decided to use the Polish version of the recording.

\section{RESULTS}

We assessed the results of the group that finished the trial (tested with the MSAQ twice). We used the T-test to see if there are any statistically significant differences in the results before and after the training on the relaxation technique. We tested both the overall scores and the four factors that the test has: Gastrointestinal, Central, Sopite-related and Peripheral. Statistically significant differences in the overall score were found $(0,0054 p<0,05)$ as in some of the factors: Central $(0,015 p<0,05)$ and Sopite-related $(0,035 p<0,05)$. Both the remaining factors exhibited a statistical trend. All the raw scores: overall and factoral ones were lower in the second testing in comparison with the first one.

\section{CONCLUSION}

The results show that there is a statistically significant difference between some of the factors measuring the severity of motion sickness after training with the relaxation technique. What it shows us is that a simple, low time-consuming and inexpensive method may be used along other training options to further expand pilots' skills. The results shown were gathered from a small group of subjects that definitely should be broadened. However, the promising results should encourage to continue testing such methods on bigger groups of pilots in the near future as we already intend to do.

\section{AUTHORS' DECLARATION:}

Study Design: Michał Janewicz, Krzysztof Kowalczuk; Data Collection and Analysis: Krzysztof Kowalczuk, Michał Janewicz, Zofia Przymus, Tadeusz Grzeszuk, Robert Kilian, Stefan P. Gaździński; Manuscript Preparation: Krzysztof Kowalczuk, Michał Janewicz, Zofia Przymus, Stefan P. Gaździński; Funds Collection: Krzysztof Kowalczuk. The Authors declare that there is no conflict of interest.

\section{REFERENCES}

1. Ernsting J, Nicholson AN, Rainford DJ. Aviation Medicine. CRC Press 1999.

2. Golding JF. Motion sickness susceptibility. Autonomic Neuroscience. 2006; 129(1-2):67-76.

3. Kennedy RS, Fowlkes JE, Berbaum KS, Lilenthal MG. Use of a motion sickness history questionnaire for prediction of simulator sickness. Aviation, Space, and Environmental Medicine. 1992; 63(7):588-593.

4. Kennedy RS, Lane NE. Simulator Sickness Questionnaire: An enhanced method for quantifying simulator sickness. The International Journal of Aviation Psychology. 1993; 3(3):203-220.

5. Linden W. Autogenic Training: A narrative and quantitative review of clinical outcome. Applied Psychophysiology and Biofeedback. 1994; 19(3):227-264.

Cite this article as: Kowalczuk K, Janewicz M, Przymus Z, Grzeszuk T, Kilian R, Gaździński S. The Influence of Relaxation Training Techniques on Pilots' Levels of Tolerance to Motion Sickness Symptoms During Simulated Flight - Preliminary Results. Pol J Aviat Med Bioeng Psychol 2018; 24(2): 35-37. DOI: 10.13174/pjambp.30.10.2019.05 